\title{
Comparison of phenolics, antioxidant capacity and total phenol bioaccessibility of Ribes spp. grown in Turkey
}

\author{
Hacer Eksi KARAAGAC ${ }^{1,2}$, Yasemin ŞAHAN ${ }^{3 *}$ (i)
}

\begin{abstract}
In this study, anthocyanins (13), flavanols (6), phenolic acids (10), flavonol glycosides (17), antioxidant capacity, and bioaccessible phenolic content of Ribes spp., grown in Turkey were investigated. Individual phenolic compounds were identified and quantified with LC-QTOF/MS in red and black currants and hybrid Jostaberry. Significant variations in the individual phenolic compounds could be observed between the different cultivars. In all black currant cultivars, cyanidin 3,5-di-O-glucoside was the predominant anthocyanin compounds $(p \leq 0.01)$. Cyanidin 3-O-sambubioside and cyanidin 3-O-rutinoside were detected in red currants. In all Ribes cultivars quercetin 3-O-rutinoside was the major flavonol glycoside and epigallocatechin found as the dominant flavonol compound. Rosenthal had the highest amount of total phenols, antioxidant capacity levels by DPPH and CUPRAC. Boskoop Giant characterized with the highest amount of total anthocyanin and bioaccessibility of phenolic compounds (84.27\%).
\end{abstract}

Keywords: anthocyanins; flavanols; flavonol; glycosides; jostaberry.

Practical Application: Comparison of antioxidative properties of Ripes spp. fruits for human health.

\section{Introduction}

Berries have remarked for their nutritional and bioactive properties based on good source of polyphenolic compounds (Anttonen \& Karjalainen, 2006). Many research announced that polyphenolic compounds, mostly flavonoids, and anthocyanins have amazing health-promoting properties (Kim et al., 2005). Also, epidemiological studies indicate that the consumption of anthocyanins prevents diabetes, oxidative stress, and the risk of cardiovascular disease (Zafra-Stone et al., 2007). Total phenols and total anthocyanins have been reported as the main contributors to the antioxidant effect of many berries (Tabart et al., 2006; Djordjević et al., 2010). In literature, berry flavonols such as myricetin, quercetin, kaempferol, and their derivatives concentrations show an alteration which could be explained by cultivars, their growth conditions, maturation, environmental condition and the methodological procedures applied (Mikulic-Petkovsek et al., 2012).

In the world berry production, Ribes spp., are significant currants second rate in consumer preferences immediately after strawberries (Djordjević et al., 2010). The most important Ribes species are; Ribes nigrum L., (black currants) and Ribes rubrum L., (red currant) cultivars (Djordjević et al., 2014). The main phenolic compounds reported in Ribes spp. include anthocyanins (Slimestad \& Solheim, 2002; Anttonen \& Karjalainen, 2006; Borges et al., 2010), flavonols, flavanols glycosides, and phenolic acids. These various compounds may work synergistically to promote human health.

Despite the enormous research on the total phenolic content of Ribes spp., studies investigating their bioaccessibility are scarce
(Bordonaba \& Terry, 2008; Contessa et al., 2013; Nour et al., 2013). Determination of total phenolic content bioavailability is important because only gastrointestinal sustainable phenolic can be bioaccessible for absorption (Chiang et al., 2013). Thus, the health effects of polyphenols depend on the amount consumed and on their bioavailability which appears to differ greatly. For this reason, knowledge of bioavailability is necessary for exploring the health effects of polyphenols in the fruit. Most polyphenols exist in the form of glycosides, esters, or polymers which cannot be absorbed in their native form in foods. Therefore, some polyphenols can be absorbed less efficiently and bioavailability than others, even though they are present large amounts. However, bioavailability research has practical, technical and ethical difficulties. Therefore, there is a need to develop and use in vitro models that mimic the physiological processes that occur in human digestion. (Minekus et al., 2014). Because of this, the impact of bioaccessibility on the stability of phenolic compounds has been one of the more widely investigated topics during the last decade.

This research aimed to investigate certain cultivars of Ribes species grown in Turkey for their content and composition of individual phenolic compounds including anthocyanins, flavonols, phenolic acids and flavonol glycosides, antioxidant capacities, total anthocyanin content, total polyphenolic content, and their bioaccessibility. For the detection of bioaccessibility, samples were processed by an in vitro digestive enzymatic extraction that mimics the conditions in the gastrointestinal tract. The polyphenol composition of the berry fruits has been characterized utilizing 
quadrupole time of flight mass spectrometry (LC QTOF/MS) and for other bioactive characteristics, spectrophotometric techniques were used.

\section{Materials and methods}

\subsection{Plant material}

Black currant (R. Nigrum L. cv. Boskoop Giant, Rosenthal, Goliath); red currant (R. Rubrum L. cv. Red Lake) and a cross between black currant and gooseberry (R. Nidigrolaria L. $\mathrm{cv}$. Jostaberry) fruits were harvested from Bursa, Turkey. For this study, we selected 5 fields in the same region and nearby. $25 \mathrm{~kg}$ healty fruit were harvested at commercial ripening from different parts of the field for each cultivar. The fruits were transferred to the laboratory and the moisture content of all cultivars were immediately analyzed. The samples were frozen $\left(-80^{\circ} \mathrm{C}\right)$ and stored for analyses.

\subsection{Reagents, standards and solutions}

HPLC-grade methanol, ethanol, Folin-Ciocalteu phenol reagent, formic acid, sodium chloride, sodium carbonate, sodium acetate, ammonium acetate, and copper (II) chloride were purchased from Merck (Darmstadt, Germany). HPLC-grade acetonitrile, 1,1-diphenyl-2-picrylhydrazyl (DPPH), 6-hydroxy-2,5,7,8 tetramethylchroman-2-carboxylic acid (Trolox), neocuproine, gallic acid and potassium chloride, sodium hydroxide, pepsin, bile extract, pancreatin, concentrated hydrochloric acid (37\% w/v) and concentrated sulfuric acid (95-98\%) were purchased from Sigma (St. Louis, MO, USA). The standards were used for the quantification of phenolic compounds were purchased from Extrasynthese (Genay Cedex, Francea) and Sigma-Aldrich Chemie (St. Louis, MO, USA).

\subsection{Extraction of extractable, hydrolyzable and bioaccessible fractions of phenols}

Extractable, hydrolysable, and bioaccessible fraction were extracted according to Vitali et al. (2009) with slight modifications. The extractable and hydrolyzable fraction was used for total phenolic content, antioxidant capacity, total anthocyanins, and individual phenolic compounds analysis (Vitali et al., 2009). For the determination of bioaccessible fractions, investigated Ribes varieties were processed by an in vitro digestive enzymatic extraction that mimics the conditions in the gastrointestinal tract.

\subsection{Determination of total phenolic content and antioxidant capacity}

The extractable, hydrolyzable and bioaccessible fraction of total phenolic content of fruits was determined using Folin-Ciocalteu method (Singleton et al., 1999). Gallic acid was used as a standard and the results were expressed as $\mathrm{mg} \mathrm{GAE} / \mathrm{g} \mathrm{dw}$.

Extractable and hydrolyzable extracts of investigated samples using 1,1-Diphenyl-2-picrylhydrazy Radical Scavenging, (DPPH) and Cupric Ion Reducing Antioxidant Capacity (CUPRAC) methods. DPPH method was determined by the method outlined by Brand-Williams et al. (1995) and CUPRAC method was carried out as described by Apak et al. (2004).
A calibration curve was prepared, Trolox (6-hydroxy-2,5,7,8tetramethylchromane-2-carboxylic acid) and the results were expressed as $\mu \mathrm{mol} \mathrm{TE} / \mathrm{g} \mathrm{dw}$ for each method. TPC, CUPRAC and DPPH results were calculated as the sum of extractable and hydrolyzable fraction extracts.

\subsection{Total anthocyanin}

Anthocyanin quantification was performed by the $\mathrm{pH}$-differential method (Giusti \& Wrolstad, 2001). Calculation of the anthocyanins concentration was based on a cyanidin-3-glucoside molar extinction coefficient 26,900 and a molecular mass of $449.2 \mathrm{~g} / \mathrm{mol}$. Results were expressed as milligrams of cyanidin-3-glucoside equivalents per $100 \mathrm{~g}$ of dry weight basis.

\subsection{Individual phenolic compounds}

Identification and quantification of anthocyanins and flavonols, phenolic acids and flavonol glycosides in Ribes fruits were carried out with an Agilent 6550 LC-QTOF/MS-ESI (Agilent Technologies, USA) system equipped with a Poroshell 120 EC-C 18 column $(4.6 \times 100 \mathrm{~mm}, 2.7 \mu \mathrm{m}$ film thickness $)$. For elution of these compounds, the mobile phase consisted of two solvents: formic acid $(1 \%, \mathrm{v} / \mathrm{v})$ in water $(\mathrm{A})$ and formic acid $(1 \%, \mathrm{v} / \mathrm{v})$ in acetonitrile (B). All phenolic compounds were identified using an LC-QTOF mass spectrometer with electrospray ionization (ESI) operating in the negative (non-anthocyanin phenolic compounds) and positive (for anthocyanins) ion mode. Identification of the components was carried out by comparing the retention times and MS2 fragmentation in currant samples. For all compound, MS identification was confirmed with authentic standards. The MS data are presented in Table 1. Concentrations were expressed in $\mathrm{mg} / \mathrm{kg} \mathrm{dw}$.

For non-anthocyanin and anthocyanin compounds of samples, the total run time was $20,40 \mathrm{~min}$, with $8,5 \mathrm{~min}$ of equilibration treatment performed before each analysis, the analysis was carried out using full-scan data-dependent MS2 scanning from $\mathrm{m} / \mathrm{z} 50$ to 3000,100 to 1600 , flow rate was $0.6,0.3 \mathrm{~mL} / \mathrm{min}$, column temperature was set at $40^{\circ} \mathrm{C}, 25^{\circ} \mathrm{C}$, nitrogen was used as nebulizing and drying gas at a pressure of 35,45 psi and the flow rate was adjusted to $14,12 \mathrm{~L} / \mathrm{min}$. respectively.

\subsection{Statistical analysis}

All results were expressed as mean values \pm standard deviation (SD) for six replicates. Statistical analyses were performed with JMP software, v.9.0.2 (SAS, USA). Fisher's LSD test was used to compare the means among treatments with significant differences $(p \leq 0.01)$.

\section{Results and discussion}

\subsection{Total phenolic content}

The content of extractable, hydrolyzable and bioaccessible phenolics of Ribes spp. cultivars are presented in Figure 1. Significant differences in extractable fraction, hydrolyzable fraction and total phenolic content among the different species were recorded ( $p<0.01)$. Generally, total phenolic content was in fruits vary according to numerous genetic, environmental, and technologic factors (Manach et al., 2004). 
The highest total phenolic content (123.22 mg GAE/g dw) detected in Rosenthal cultivar, followed by Boskoop giant (112.01 mg GAE/g dw). Mikulic-Petkovsek et al. (2013) recorded that total phenolic concentrations 3519.2 and $3774.1 \mathrm{mg} \mathrm{GAE} / \mathrm{kg}$ $\mathrm{fw}$ for Goliath and Rosenthal, respectively. Our finding higher than these results for both varieties, but Mikulic-Petkovsek et al.
(2015) 4525.26-6803.41 mg GAE/100 g fw of total phenolic content detected in Rosenthal varieties, which is higher than our findings.

Red Lake cultivar showed the highest content of hydrolyzable phenols, while the lowest was recorded in hybrid Jostaberry

Table 1. Tentative identification of phenolic compounds in Ribes spp. using LC-QTOF/MS-ESI.

\begin{tabular}{|c|c|c|c|c|c|c|}
\hline Compound Name & $\mathrm{m} / \mathrm{z}$ & $\begin{array}{c}\text { Detected } \\
\text { Mass }\end{array}$ & RT & $\begin{array}{c}\text { Deviation } \\
(\mathrm{ppm})\end{array}$ & Mass & MS/MS \\
\hline Cyanidin 3-O-glucoside & 449.1084 & 449.1060 & 9.70 & 5.34 & 484.0772 & $287.0631 / 139.0392$ \\
\hline Cyanidin 3-O-sambubioside & 581.1502 & 581.1524 & 9.54 & -3.79 & 616.1190 & $287.0621 / 118.0894$ \\
\hline Cyanidin 3-O-rutinoside & 595.1663 & 595.1647 & 10.1 & 2.69 & 630.1351 & $449.1126 / 287.0644$ \\
\hline Cyanidin 3-O-galactoside & 449.1084 & 449.1068 & 9.50 & 3.56 & 484.0772 & $287.0641 / 137.0269$ \\
\hline Cyanidin 3,5-di-O-glucoside & 611.1612 & 611.1618 & $8 ., 29$ & -0.98 & 646.1301 & $449.1206 / 287.0639$ \\
\hline Peonidin 3-O-rutinoside & 609.1820 & 609.1841 & 11.36 & -3.45 & 644.1508 & $301.0787 / 121.0334$ \\
\hline Peonidin 3-O-glucoside & 463.1241 & 463.1258 & 11.49 & -3.67 & 498.0928 & $301.0775 / 89.0622$ \\
\hline Pelargonidin 3,5-di-O-glucoside & 596.1670 & & & & & $287.0617 / 111.3086$ \\
\hline Pelargonidin 3-O-rutinoside & 579.1741 & 579.1741 & 10.34 & 0.00 & 579.171 & $433.1217 / 271.0684$ \\
\hline Petunidin 3-O-glucoside & 479.1241 & 479.1241 & 10.84 & 0.00 & 479.1190 & $317.0741 / 137.0277$ \\
\hline Delphinidin 3-O-rutinoside & 611.1613 & 611.1623 & 9.99 & -1.64 & 646.1301 & $287.0643 / 137.0263$ \\
\hline Delphinidin 3-O-glucoside & 465.1077 & 465.1077 & 8.89 & 0.00 & 465.1027 & $303.0597 / 273.0463$ \\
\hline Malvidin 3-O-glucoside & 493.1346 & 493.1361 & 11.93 & -3.04 & 528.1031 & $331.0914 / 85.0316$ \\
\hline Catechin & 289.0711 & 289.0712 & 4.49 & -0.35 & 290.079 & $289.0712 / 245.0819 / 205.0506$ \\
\hline Epigallocatechin & 305.0660 & 305.0665 & 3.83 & -1.64 & 306.0739 & $125.0247 / 160.8413 / 197.9165$ \\
\hline Epicatechin & 289.0711 & 289.0713 & 5.07 & -0.69 & 290.079 & $245.08140 / 205.0549 / 125.0244$ \\
\hline Epigallocatechin gallate & 457.0770 & 457.0764 & 5.09 & 1.31 & 458.0849 & $125.0244 / 169.0142 / 161.0244$ \\
\hline Epicatechin gallate & 441.0820 & 441.0816 & 5.60 & 0.91 & 442.0899 & $169.0147 / 125.0252 / 289.0719$ \\
\hline Catechin & 289.0711 & 289.0712 & 4.49 & -0.35 & 290.079 & $289.0712 / 245.0819 / 205.0506$ \\
\hline Chlorogenic acid & 353.0871 & 353.0869 & 4.34 & 0.57 & 354.0950 & $191.0561 / 353.0878 / 161.0244$ \\
\hline 2-Hydroxybenzoic acid & 137.0237 & 137.0242 & 6.64 & -3.65 & 138.0316 & $93.0368 / 65.0421 / 75.0240$ \\
\hline Gentisic acid & 153.0187 & 153.0181 & 4.86 & 3.92 & 154.0266 & $108.0216 / 53.0396$ \\
\hline 4-hydroxy benzoic acid & 137.0237 & 137.0240 & 4.83 & -2.19 & 138.0316 & $137.0237 / 108.0219$ \\
\hline Ellagic acid & 300.9983 & 300.9985 & 5.51 & -0.66 & 302.0062 & $257.0087 / 229.2126 / 185.0235$ \\
\hline Caffeic acid & 179.0343 & 179.0351 & 5.13 & -4.47 & 180.0422 & $134.0373 / 89.0396 / 135.0451$ \\
\hline Vanillic acid & 167.0343 & 167.0345 & 5.14 & -1.20 & 168.0422 & $135.0460 / 108.0228$ \\
\hline Gallic acid & 169.0136 & 169.0143 & 2.32 & -4.14 & 170.0215 & $125.0253 / 147.8918 / 107.0149$ \\
\hline Protocatechuic acid & 153.0187 & 153.0191 & 3.53 & -2.61 & 154.0266 & $109.0297 / 141.8689 / 118.0304$ \\
\hline P-Cumaric acid & 163.0394 & 163.0394 & 5.68 & 0.00 & 164.0473 & $119.0513 / 145.0298$ \\
\hline Myricetin 3-O-glucoside & 479.0821 & 479.0818 & 5.20 & 0.63 & 480.0900 & $317.6521 / 137.0267$ \\
\hline Myricetin 3-O-galactoside & 479.0821 & 479.0818 & 5.16 & 0.63 & 480.0900 & $317.6521 / 137.0268$ \\
\hline Myricetin 3-O-rhamnoside & 463.0875 & 463.0872 & 5.44 & 0.65 & 464.0954 & $463.0872 / 317.0598$ \\
\hline Quercetin 3-glucoside & 463.0875 & 463.0869 & 5.46 & 1.30 & 464.0954 & $463.0869 / 301.9782$ \\
\hline Quercetin 3-D-galactoside & 463.0875 & 463.0868 & 5.43 & 1.51 & 464.0954 & $463.0868 / 301.9782$ \\
\hline Quercetin & 301.0298 & 301.0338 & 8.50 & -13.29 & 301.0298 & $151.0045 / 107.0150 / 178.9991$ \\
\hline Quercetin 3-O-rhamnoside & 301.0347 & 301.0351 & 6.53 & -1.33 & 302.0426 & 301.0351 \\
\hline Quercetin 3-D-xyloside & 433.0771 & 433.0761 & 5.58 & 2.31 & 434.0850 & $433.0761 / 301.347$ \\
\hline Quercetin 3-O-rutinoside hyd. & 609.1451 & 609.1448 & 5.31 & 0.49 & 610.1530 & $300.0290 / 463.0881 / 343.0463$ \\
\hline Isorhamnetin & 315.0503 & 315.0513 & 7.06 & -3.17 & 316.0582 & $300.0277 / 151.0033 / 107.0142$ \\
\hline Isorhamnetin 3-O-rutinoside & 623.1611 & 623.1626 & 7.70 & -2.41 & 624.1690 & $477.1087 / 315.2321$ \\
\hline Isorhamnetin 3-O-glucoside & 477.1031 & 477.1025 & 5.69 & 1.26 & 478.1110 & $477.1025 / 315.0904$ \\
\hline Syringetin 3 glucoside & 507.1138 & 507.1131 & 5.67 & 1.38 & 508.1217 & $507.1131 / 345.0301$ \\
\hline Kaempferol & 285.0398 & 285.0404 & 7.05 & -2.10 & 286.0477 & $152.0687 / 67.1025$ \\
\hline Kaempferol 3-B-D glucoside & 447.0931 & 447.0952 & 11.10 & -4.70 & 448.1010 & $447.0938 / 284.0336$ \\
\hline Kaempferol 3-O-D-galactoside & 447.0931 & 447.0938 & 13.15 & -1.57 & 448.1010 & $284.0336 / 169.0142$ \\
\hline Kaempferol 3-B-D rutinoside & 593.1501 & 593.1482 & 19.46 & 3.20 & 594.1580 & $285.0412 / 447.0897 / 327.0504$ \\
\hline
\end{tabular}

$\mathrm{m} / \mathrm{z}$ : mass-to-charge ratio; RT: retention time; MS: mass spectrum. 
cultivar (Table 2). In terms of total phenolic content, Red lake cultivar contained the lowest amount among all species. In literatüre, total phenolic content was found that $0.418 \mathrm{~g}$ GAE $100 \mathrm{~g}^{-1} \mathrm{fw}$ (Plessi et al., 2007) and 1115-1193 mg GAE 100/g (Pantelidis et al., 2007) in red lake cultivar; and 18.94-35.85 mg GAE/g dw (Woznicki et al., 2015) and $251.9 \mathrm{mg} \mathrm{GAE} / 100 \mathrm{~g} \mathrm{fw}$ (Djordjević et al., 2014) in black currant cultivar. In comparison with those researches, the results obtained by our study of total phenolic content in both red and black currants are much higher than other results.

In Jostaberry relatively high amount of total phenolic content was detected (1809.24 mg GAE/kg fw), the amount of this cultivar was following by the report of Mikulic-Petkovsek et al. (2015) results. Bioaccessibility of total phenolic content in investigated Ribes spp. cultivars ranged from $69.91 \%$ to $89.07 \%$. Data on bioaccessibility of total phenolic content from Ribes spp. are quite limited.

\subsection{Antioxidant capacity}

The antioxidant capacities of the samples were determined by DPPH and CUPRAC methods. In the comparison of the levels of DPPH and CUPRAC antioxidant capacities among Ribes varieties, differences were observed $(p<0.01)$ (Table 2$)$. According to our results, Ribes nigrum (black currant) varieties had the highest antioxidant capacity among all Ribes species.
High antioxidant potential of black currant has been reported previously (Ehala et al., 2005) and this property correlated with its high content of phenolic compounds.

DPPH values of Ribes species ranged from 68.61 to $181.38 \mu \mathrm{mol} \mathrm{TE} / \mathrm{g} \mathrm{dw}$. The highest DPPH antioxidant capacity was detected in Boskoop giant followed by Rosenthal cultivar, whereas the lowest value was detected in Red lake cultivar.

These levels are comparable with Mikulic-Petkovsek et al. (2015) reported that DPPH level ranged from $161.65-365.50 \mathrm{mg}$ AAE 100/g fw for Rosenthal, 59.98-103.81 mg AAE 100/g fw for red currant and 33.93-103.02 mg AAE 100/g fw for Jostaberry cultivars, in three maturity stages.

The values of CUPRAC were in the range of $299.64 \mu \mathrm{mol} \mathrm{TE} / \mathrm{g} \mathrm{dw}$ (Red lake) to $914.31 \mu \mathrm{mol} \mathrm{TE} / \mathrm{g} \mathrm{dw}$ (Rosenthal) in Ribes species. Following the Rosenthal, the highest level of CUPRAC was detected in Boskoop giant and Goliath cultivar, respectively.

\subsection{Total anthocyanin content}

Significant variations $(p<0.01)$ in the total anthocyanin content could be observed between the different currant varieties (Table 2). These variations could be from the cultivars, maturation and agricultural treatment, as well as environmental and weather conditions.

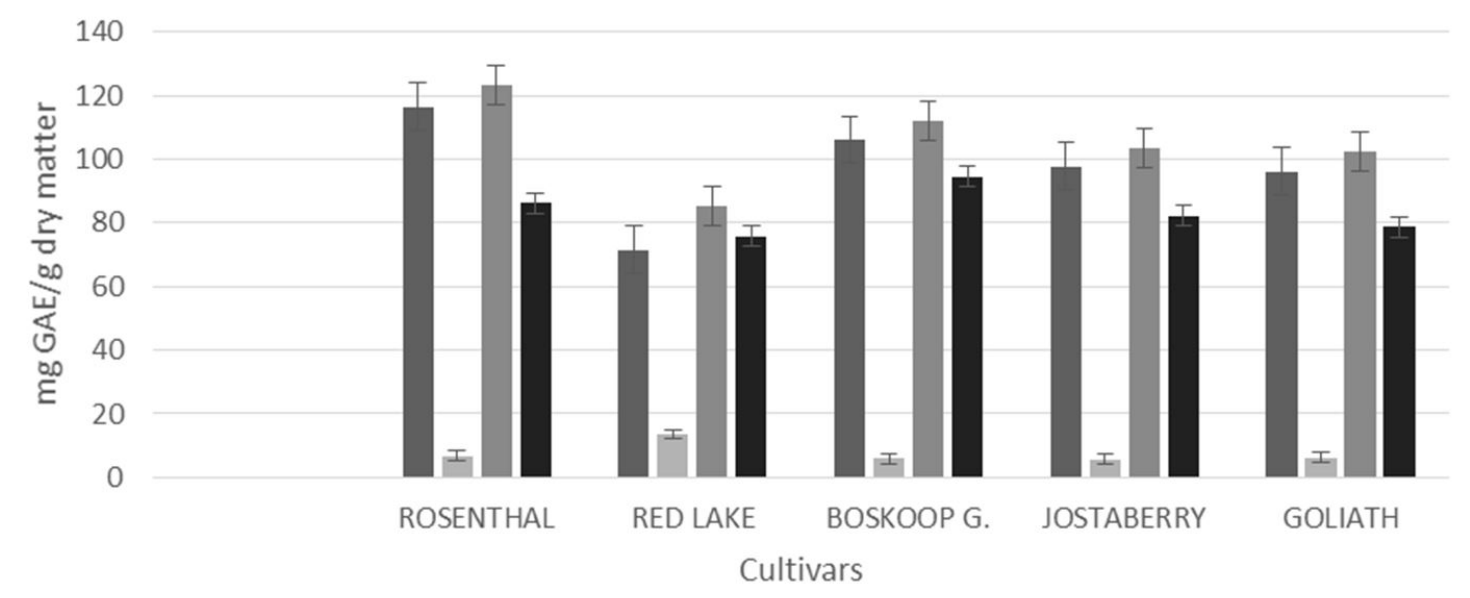

\section{Extractable fraction $\quad$ Hydrolyzable fraction $\quad$ Total phenolic content Bioaccessible fractions}

Figure 1. The content of extractable, hydrolysable and bioaccessible fraction of phenolic content of Ribes spp. Data are presented as Mean \pm SD $(n=6)$.

Table 2. Total anthocyanin and antioxidant capacity of Ribes spp.

\begin{tabular}{|c|c|c|c|c|c|}
\hline & Rosenthal & Red Lake & Boskoop G. & Jostaberry & Goliath \\
\hline $\begin{array}{l}\text { Total anthocyanin } \\
\text { (mg cy-3 glu/100 g) }\end{array}$ & $1586.76 \pm 60.07^{b}$ & $127.13 \pm 6.83^{e}$ & $1777.73 \pm 90.07^{a}$ & $349.89 \pm 32.54^{\mathrm{d}}$ & $1101.07 \pm 60.07^{\circ}$ \\
\hline \multicolumn{6}{|l|}{$\begin{array}{l}\text { Antioxidant capacity } \\
(\mu \mathrm{mol} \mathrm{TE} / \mathrm{g})\end{array}$} \\
\hline DPPH & $75.79 \pm 6.51^{\mathrm{cd}}$ & $181.38 \pm 4.11^{\mathrm{a}}$ & $68.61 \pm 4.85^{\mathrm{d}}$ & $165.35 \pm 4.88^{\mathrm{b}}$ & $84.15 \pm 6.27^{c}$ \\
\hline CUPRAC & $914.31 \pm 62.31^{\mathrm{a}}$ & $299.64 \pm 7.85^{c}$ & $847.17 \pm 67.12^{\mathrm{a}}$ & $369.33 \pm 29.99^{c}$ & $638.15 \pm 59.90^{\mathrm{b}}$ \\
\hline
\end{tabular}


Boskoop giant cultivar had the highest total anthocyanin content (1777.73 mg cy-3 glu/100 g dw), while the lowest content (127.13 mg cy-3 glu/100 g dw) was recorded in Red lake cultivar. Following the Boskoop giant, Rosenthal and Goliath varieties contained a relatively high amount of total anthocyanin content. The similar result was found by Anttonen \& Karjalainen (2006), Plessi et al. (2007), and Mikulic-Petkovsek et al. (2015) however, our results of total anthocyanin content for black currant were higher than Slimestad \& Solheim (2002) (250 mg cy-3 glu/100 g fw), Bordonaba \& Terry (2008) (83-199 mg/100 g fw), Contessa et al. (2013) (224.79 mg cy-3 glu/100 g fw), Nour et al. (2013) (116.7 to $287.78 \mathrm{mg} / 100 \mathrm{~g}$ ), Woznicki et al. (2015) (7.23-12.65 mg cy-3 glu/100 g dw) and Paunović et al. (2017) (207.5 to $372.9 \mathrm{mg}$ cy-3 glu/100 g) results. In literature, Plessi et al. (2007), Pantelidis et al. (2007), Borges et al. (2010), Djordjević et al. (2014) and Mattila et al. (2016) reported that the amounts of total anthocyanins in red currants average $2 \mathrm{mg}$ cy-3 glu/100 g fw, 7.5-7.8 mg cy-3 glu/100 g fw, $14.7 \mathrm{mg}$ cy-3 glu/100 g fw, $11.9 \mathrm{mg} / 100 \mathrm{~g} \mathrm{fw}$ and $138-462 \mathrm{mg} / 100 \mathrm{~g} \mathrm{dw}$, respectively. In addition, according to Mikulic-Petkovsek et al. (2015) research Jostaberry fruits contained between $265.38-734.72 \mathrm{mg} / \mathrm{kg}$ fw total anthocyanin in three maturity stages. Our results for the same varieties were between the authors' findings.

\subsection{Phenolic profile}

Phenolic compounds of Ribes species include anthocyanins, phenolic acids and flavonols being the dominant group (Anttonen $\&$ Karjalainen, 2006). Significant variations $(p \leq 0.01)$ in the individual phenolic compounds could be observed among the different Ribes species cultivars (Table 3 and 4). These differences among fruits for the quantity and the composition of phenolic compounds might be explained by several factors such as the genotype, phenotypic differences, species, cultivar properties, and growing condition (Strack, 1997).

\subsection{Anthocyanin compounds}

Significant differences in individual anthocyanin content among the different Ribes species were recorded $(p \leq 0.01)$. Anthocyanins belonging to the group of flavonoids were the major phenolic group in black currant as they accounted 94.48-97.96\% of total phenolic compounds analyzed, whereas the quite low proportion of anthocyanins were recorded in red currant $(22.13 \%)$ (Table 3 ). According to our results, cyanidin 3,5 di-glucoside was the major anthocyanin in all black currant varieties (3339.19-5447.18 mg/kg dw), followed by delphinidin 3-rutinoside, cyanidin 3-rutinoside and delphinidin 3-glucoside and Boskoop giant cultivar had the highest amount. In the comparison of order, our findings were similar with Slimestad \& Solheim (2002), Rubinskiene et al. (2005), Anttonen \& Karjalainen (2006), Borges et al. (2010), Gavrilova et al. (2011), MikulicPetkovsek et al. (2016), except of cyanidin 3,5 di-glucoside which is determined as the predominant anthocyanin compound of black currants.

Red lake cultivar contained only cyanidin glycosides ( $119.66 \mathrm{mg} / \mathrm{kg} \mathrm{dw}$ of cyanidin 3-O-sambubioside and $125.96 \mathrm{mg} / \mathrm{kg} \mathrm{dw}$ of cyanidin 3-O-rutinoside) with the lowest content of total amount of individual anthocyanin $(245.62 \mathrm{mg} / \mathrm{kg} \mathrm{dw})$. Especially, cyanidin $3 \mathrm{O}$-sambubioside is determined only in the Red lake cultivar, appear to be characteristic for red currant and probably contribute to their color. In literatüre, cyanidin 3-O-(2"-xyl) rutinoside, cyanidin 3-sambubioside and cyanidin 3-rutinoside were determined as the major anthocyanin compounds in red currants, respectively (Borges et al., 2010), while other authors reported cyanidin 3-rutinoside as the dominant anthocyanin in red currant cultivars (Mikulic-Petkovsek et al., 2015).

Although Jostaberry had the same anthocyanin compounds as black currant, the concentrations of these anthocyanins were

Table 3. Characterization of anthocyanin and phenolic acids determined in Ribes spp.

\begin{tabular}{|c|c|c|c|c|c|}
\hline Compound Name & Rosenthal & Red Lake & Boskoop G. & Jostaberry & Goliath \\
\hline \multicolumn{6}{|l|}{ Anthociyanins (mg/kg) } \\
\hline Cyanidin 3-O-glucoside & $25.87 \pm 1.63^{c}$ & $\mathrm{Nd}$ & $165.38 \pm 14.07^{\mathrm{b}}$ & $46.04 \pm 3.29^{c}$ & $402.17 \pm 28.79^{a}$ \\
\hline Cyanidin 3-O-sambubioside & $\mathrm{Nd}$ & $119.66 \pm 3.09^{a}$ & $\mathrm{Nd}$ & $\mathrm{Nd}$ & $\mathrm{Nd}$ \\
\hline Cyanidin 3-O-rutinoside & $2177.97 \pm 82.10^{c}$ & $125.96 \pm 9.71^{\mathrm{e}}$ & $3333.56 \pm 63.93^{\mathrm{a}}$ & $1380.81 \pm 62.06^{\mathrm{d}}$ & $2627.47 \pm 52.79^{b}$ \\
\hline Cyanidin 3-O-galactoside & $172.08 \pm 16.77^{b}$ & $\mathrm{Nd}$ & $115.83 \pm 8.67^{c}$ & $81.13 \pm 8.44^{\mathrm{d}}$ & $303.0 \pm 21.05^{\mathrm{a}}$ \\
\hline Cyanidin 3,5-di-O-glucoside & $4038.12 \pm 98.51^{b}$ & $\mathrm{Nd}$ & $5447.18 \pm 87.71^{\mathrm{a}}$ & $665.35 \pm 28.83^{\mathrm{d}}$ & $3339.19 \pm 157.33^{c}$ \\
\hline Delphinidin 3-O-rutinoside & $3518.01 \pm 88.97^{\mathrm{b}}$ & $\mathrm{Nd}$ & $4652.73 \pm 90.46^{\mathrm{a}}$ & $602.78 \pm 12.39^{d}$ & $2913.42 \pm 96.84^{c}$ \\
\hline Delphinidin 3-O-glucoside & $357.48 \pm 20.59^{c}$ & $\mathrm{Nd}$ & $789.44 \pm 22.75^{b}$ & $83.06 \pm 12.41^{\mathrm{d}}$ & $987.86 \pm 74.95^{\mathrm{a}}$ \\
\hline \multicolumn{6}{|l|}{ Phenolic acids (mg/kg) } \\
\hline Chlorogenic acid & $\mathrm{Nd}$ & $1.79 \pm 0.11^{\mathrm{c}}$ & $4.45 \pm 0.14^{\mathrm{a}}$ & $1.15 \pm 0.04^{\mathrm{d}}$ & $4.17 \pm 0.28^{\mathrm{b}}$ \\
\hline 2-Hydroxybenzoic acid & $2.47 \pm 0.38^{c}$ & $14.36 \pm 1.70^{\mathrm{b}}$ & $11.12 \pm 1.19^{\mathrm{b}}$ & $14.72 \pm 2.45^{\mathrm{b}}$ & $42.94 \pm 5.19^{\mathrm{a}}$ \\
\hline Gentisic acid & $\mathrm{Nd}$ & $3.60 \pm 0.21^{\mathrm{a}}$ & $0.69 \pm 0.22^{\mathrm{d}}$ & $2.69 \pm 0.11^{\mathrm{b}}$ & $1.42 \pm 0.13^{c}$ \\
\hline 4-hydroxy benzoic acid & $0.15 \pm 0.00^{\mathrm{b}}$ & $0.11 \pm 0.01^{\mathrm{c}}$ & $0.06 \pm 0.01^{\mathrm{d}}$ & $0.20 \pm 0.03^{\mathrm{a}}$ & $0.17 \pm 0.02^{\mathrm{ab}}$ \\
\hline Ellagic acid & $\mathrm{Nd}$ & $0.83 \pm 0.12^{\mathrm{b}}$ & $0.65 \pm 0.05^{c}$ & $1.78 \pm 0.17^{\mathrm{a}}$ & $\mathrm{Nd}$ \\
\hline Caffeic acid & $2.35 \pm 0.13^{\mathrm{d}}$ & $7.92 \pm 0.25^{\mathrm{a}}$ & $2.80 \pm 0.17^{c}$ & $8.02 \pm 0.19^{a}$ & $6.14 \pm 0.35^{\mathrm{b}}$ \\
\hline Vanillic acid & $52.28 \pm 1.89^{\mathrm{a}}$ & $16.57 \pm 1.17^{\mathrm{c}}$ & $17.19 \pm 1.47^{c}$ & $17.30 \pm 0.79^{c}$ & $34.87 \pm 3.48^{\mathrm{b}}$ \\
\hline Gallic acid & $0.07 \pm 0.00^{\mathrm{e}}$ & $0.27 \pm 0.03^{\mathrm{a}}$ & $0.16 \pm 0.02^{c}$ & $0.12 \pm 0.02^{\mathrm{d}}$ & $0.21 \pm 0.03^{\mathrm{b}}$ \\
\hline Protocatechuic acid & $5.72 \pm 0.20^{\mathrm{d}}$ & $17.49 \pm 1.10^{\mathrm{b}}$ & $1.16 \pm 0.31^{\mathrm{e}}$ & $14.03 \pm 1.64^{\mathrm{c}}$ & $21.65 \pm 3.33^{\mathrm{a}}$ \\
\hline P-Coumaric acid & $0.58 \pm 0.09^{\mathrm{d}}$ & $3.01 \pm 0.07^{\mathrm{a}}$ & $1.38 \pm 0.08^{c}$ & $1.51 \pm 0.06^{c}$ & $2.82 \pm 0.13^{\mathrm{b}}$ \\
\hline
\end{tabular}

Results are expressed on a dry weight basis in $\mathrm{mg} / \mathrm{kg}$ and are given as mean \pm standard deviations $(\mathrm{n}=6)$ with different letter $(\mathrm{a}$-e) in the same line are significantly different $(p \leq 0.01)$. Nd: not detected. 
Table 4. Individual quantities of flavanol and flavonol glycosides determined in Ribes fruits.

\begin{tabular}{|c|c|c|c|c|c|}
\hline Compound Name & Rosenthal & Red Lake & Boskoop G. & Jostaberry & Goliath \\
\hline \multicolumn{6}{|l|}{ Flavanol compounds $(\mathrm{mg} / \mathrm{kg})$} \\
\hline Catechin & $25.14 \pm 2.68^{\mathrm{b}}$ & $45.58 \pm 2.03^{\mathrm{a}}$ & $26.36 \pm 3.49^{b}$ & $12.93 \pm 0.56^{\mathrm{d}}$ & $22.53 \pm 0.51^{\mathrm{c}}$ \\
\hline Epigallocatechin & $11.26 \pm 0.97^{\mathrm{d}}$ & $256.13 \pm 17.34^{\mathrm{a}}$ & $158.11 \pm 14.24^{\mathrm{c}}$ & $195.93 \pm 15.34^{\mathrm{b}}$ & $150.11 \pm 7.44^{\circ}$ \\
\hline Epicatechin & $4.18 \pm 0.09^{\mathrm{d}}$ & $18.48 \pm 0.60^{\mathrm{a}}$ & $14.05 \pm 2.00^{\mathrm{bc}}$ & $16.01 \pm 1.30^{\mathrm{b}}$ & $12.38 \pm 1.40^{\circ}$ \\
\hline Epigallocatechin gallate & $1.27 \pm 0.16^{\mathrm{b}}$ & $0.34 \pm 0.03^{\mathrm{d}}$ & $1.66 \pm 0.09^{\mathrm{a}}$ & $0.19 \pm 0.03^{\mathrm{e}}$ & $0.85 \pm 0.04^{\mathrm{c}}$ \\
\hline Epicatechin gallate & $0.05 \pm 0.00^{\mathrm{b}}$ & $0.12 \pm 0.00^{\mathrm{a}}$ & $0.13 \pm 0.03^{\mathrm{a}}$ & $0.11 \pm 0.00^{\mathrm{a}}$ & $0.13 \pm 0.04^{\mathrm{a}}$ \\
\hline \multicolumn{6}{|l|}{ Flavonol glycosides $(\mathrm{mg} / \mathrm{kg})$} \\
\hline Myricetin 3-O-glucoside & $0.85 \pm 0.05^{\mathrm{e}}$ & $153.99 \pm 3.52^{\mathrm{a}}$ & $131.04 \pm 0.23^{c}$ & $16.40 \pm 0.90^{\mathrm{d}}$ & $140.26 \pm 4.22^{\mathrm{b}}$ \\
\hline Myricetin 3-O-rhamnoside & $0.60 \pm 0.03^{\mathrm{e}}$ & $30.78 \pm 0.46^{\mathrm{a}}$ & $9.50 \pm 0.18^{c}$ & $3.07 \pm 0.07^{\mathrm{d}}$ & $25.39 \pm 1.08^{\mathrm{b}}$ \\
\hline Quercetin 3 O-rhamnoside & $3.54 \pm 0.08^{\mathrm{b}}$ & $\mathrm{Nd}$ & $3.66 \pm 0.09^{\mathrm{b}}$ & $6.69 \pm 0.35^{\mathrm{a}}$ & $\mathrm{Nd}$ \\
\hline Quercetin 3 D-xyloside & $0.93 \pm 0.07^{c}$ & $0.64 \pm 0.05^{c}$ & $1.23 \pm 0.17^{\mathrm{c}}$ & $5.81 \pm 0.49^{\mathrm{b}}$ & $11.86 \pm 1.23^{\mathrm{a}}$ \\
\hline Quercetin 3-O-rutinoside & $105.64 \pm 5.68^{\mathrm{e}}$ & $257.42 \pm 6.56^{\mathrm{a}}$ & $180.26 \pm 3.62^{\mathrm{b}}$ & $160.82 \pm 6.63^{c}$ & $129.63 \pm 2.42$ \\
\hline Isorhamnetin & $0.01 \pm 0.00^{\mathrm{e}}$ & $0.78 \pm 0.03^{\mathrm{a}}$ & $0.19 \pm 0.03^{c}$ & $0.61 \pm 0.05^{\mathrm{b}}$ & $0.12 \pm 0.01^{\mathrm{c}}$ \\
\hline Isorhamnetin 3-O-rutinoside & $\mathrm{Nd}$ & $4.0 \pm 0.21^{\mathrm{b}}$ & $2.29 \pm 0.07^{\mathrm{c}}$ & $7.57 \pm 0.36^{\mathrm{a}}$ & $1.40 \pm 0.17^{\mathrm{c}}$ \\
\hline Isorhamnetin 3-O-glucoside & $\mathrm{Nd}$ & $1.67 \pm 0.06^{\mathrm{a}}$ & $1.49 \pm 0.09^{\mathrm{a}}$ & $0.69 \pm 0.02^{c}$ & $0.95 \pm 0.21^{\mathrm{b}}$ \\
\hline Syringetin 3 glucoside & $0.41 \pm 0.03^{c}$ & $0.89 \pm 0.07^{\mathrm{a}}$ & $0.57 \pm 0.05^{\mathrm{b}}$ & $\mathrm{Nd}$ & $0.37 \pm 0.02^{c}$ \\
\hline Kaempferol & $0.26 \pm 0.01^{\mathrm{d}}$ & $3.18 \pm 0.12^{\mathrm{b}}$ & $4.48 \pm 0.14^{\mathrm{a}}$ & $0.19 \pm 0.01^{\mathrm{d}}$ & $0.44 \pm 0.03^{c}$ \\
\hline Kaempferol 3-O-D-galactoside & $0.46 \pm 0.01^{\mathrm{d}}$ & $4.66 \pm 0.14^{\mathrm{b}}$ & $5.50 \pm 0.04^{\mathrm{a}}$ & $0.15 \pm 0.01^{\mathrm{e}}$ & $1.37 \pm 0.05^{\circ}$ \\
\hline Kaempferol 3-B-D rutinoside & $6.07 \pm 0.24^{\mathrm{d}}$ & $19.41 \pm 1.02^{\mathrm{b}}$ & $15.18 \pm 0.50^{c}$ & $31.13 \pm 3.05^{\mathrm{a}}$ & $4.65 \pm 0.35^{\circ}$ \\
\hline
\end{tabular}

Results are expressed on a dry weight basis in $\mathrm{mg} / \mathrm{kg}$ and are given as mean \pm standard deviations $(\mathrm{n}=6)$ with different letter (a-e) in the same line are significantly different $(p \leq 0.01)$. Nd: not detected.

low. In Jostaberry the prevailing anthocyanins had cyanidin glycosides yielding dark blue or almost black fruit color. Cyanidin 3-O-rutinoside was found as the major anthocyanin compound, followed by cyanidin 3,5-di-O-glucoside and delphinidin 3-O-rutinoside.

\subsection{Phenolic acid compounds}

Individual phenolic acid compounds levels changed significantly in tested samples (Table 3 ). In genaral, vanillic acid, 2-hydroxybenzoic acid and protocatechuic acid were abundant in all Ribes species. The highest concentration of 2-hydroxybenzoic acid and protocatechuic acid were detected in Goliath cultivar ( $42.94 \mathrm{mg} / \mathrm{kg} \mathrm{dw}$ and $21.65 \mathrm{mg} / \mathrm{kg} \mathrm{dw}$, respectively) and vanillic acid in Rosenthal cultivar ( $52.28 \mathrm{mg} / \mathrm{kg} \mathrm{dw}$ ). In total, the highest total individual phenolic acid was found in Goliath cultivar, while the lowest content was determined in Boskoop giant cultivar. Ellagic acid, 4-hydroxybenzoic acid and gallic acid were determined as the lowest phenolic acid compounds in all cultivar.

In literature, it was found that the differences for concentration of phenolic acid compounds among the Ribes species (Gavrilova et al., 2011; Mikulic-Petkovsek et al., 2015). It might be that cultivar, origin of the fruit, maturation period, agricultural teratment and growing condition caused these differences.

\subsection{Flavanol compounds}

The mean ( \pm standard deviation) and range of the concentrations of individual flavanol compounds in Ribes species are given in Table 4 . In statistical evaluation, it was found that the differences were important $(p \leq 0.01)$ among cultivars. Red lake cultivar was the richest species in terms of the total flavonol content $(320.65 \mathrm{mg} / \mathrm{kg} \mathrm{dw})$, followed by
Jostaberry cultivar $(225.16 \mathrm{mg} / \mathrm{kg} \mathrm{dw})$. Red lake, Jostaberry, Boskoop giant and Goliath cultivars contained a high amount of epigallocathechin. After epigallocatechin, catechin, and epicatechin were the abundant flavonol compounds in all cultivars. The common flavonols present in different parts of black currant plants are epigallocatechin, gallocatechin, catechin, epicatechin and epigallocatechin gallate (Tabart et al., 2011) and epigallocatechin concentration in black currant were in the range of 5.86-5.95 mg/100 g fw, $27.9 \mathrm{mg} / \mathrm{kg}$ fw in Goliath and $36.3 \mathrm{mg} / \mathrm{kg} \mathrm{fw}$ in Rosenthal cultivar (Gavrilova et al., 2011; Mikulic-Petkovsek et al., 2015; Mikulic-Petkovsek et al., 2016). When these flavanol levels were compared with the results obtained in this study, epigallocatechin concentration in black currant was different; however different growing condition, maturation, and cultivar were examined.

\subsection{Flavonol glycoside compounds}

Six (6) glycosides from the group of quercetin derivatives, three (3) glycosides from the group of myricetin, three (3) glycosides from the group of isorhamnetin, four (4) glycosides from the group of kaempferol and one (1) glycoside from the group of syringetin have been investigated in our study. Significant variations in flavonol glycoside compounds could be observed between the different Ribes species (Table 4). Myricetin 3-O-galactoside, quercetin 3-glucoside, quercetin 3-D-galactoside, quercetin, kaempferol 3-B-D glucoside compounds were not detected any Ribes species. The amount of isorhamnetin, isorhamnetin 3-O-glucoside, and syringetin-3-glucoside were quite low in all species.

Quercetin 3-O-rutinoside concentration ranged from 105.64 to $257.42 \mathrm{mg} / \mathrm{kg} \mathrm{dw}$ was the major flavonol glucoside compound in all Ribes species, except Goliath cultivar. Quercetin 
glycosides represented the main portion of total flavonols for Jostaberry (60.69\%), Red lake (54.05\%), Rosenthal (92.72\%) and Boskoop Giant (52.10\%) cultivars, whereas myricetin glycosides represented the main portion of total flavonols for Goliath (52.35\%) cultivar. In previous studies, quercetin glycosides have been reported to be the dominant flavonol in black currants varieties (Mikulic-Petkovsek et al., 2016), while Mattila et al. (2016) reported myricetin glycosides as the main flavonol. These difference may be due to the difficulty in quantifying myricetin, as this flavonol is unstable and sensitive to interference from other compounds (Justesen et al., 1998). Gavrilova et al. (2011) reported that quercetin 3-O-rutinoside level found between $0.47-1.89 \mathrm{mg} / 100 \mathrm{~g}$ fw in red currants and $4.24-4.58 \mathrm{mg} / 100 \mathrm{~g} \mathrm{fw}$ in black currants, these findings were in line with our results. Myricetin has been quantified in all Ribes cultivars analyzed; however, levels of these compound in red currant were significantly higher than black currant cultivars. The third prevailing flavonol compounds belonged to the group of kaempferol derivatives $(6.46-31.47 \mathrm{mg} / \mathrm{kg} \mathrm{dw})$ in all species. In our research, the highest levels of total kaempferol compounds have been measured in the Jostaberry $(31.47 \mathrm{mg} / \mathrm{kg} \mathrm{dw})$, followed by Red lake $(27.25 \mathrm{mg} / \mathrm{kg} \mathrm{dw})$, on the contrary, Paunović et al. (2017) reported the amount of kaempferol was very low in all cultivars.

Red lake cultivar had the highest amount of total individual flavonol glycoside and especially quercetin $(258.06 \mathrm{mg} / \mathrm{kg} \mathrm{dw})$ and myricetin $(184.77 \mathrm{mg} / \mathrm{kg} \mathrm{dw})$ derivatives were present in high amount and followed by kaempferol derivatives $(27.25 \mathrm{mg} / \mathrm{kg} \mathrm{dw})$ in this cultivar. For Jostaberry, total individual flavonol glycoside concentration was found $233.13 \mathrm{mg} / \mathrm{kg} \mathrm{dw}$. Quercetin 3-O-rutinoside level was the most abundant among the flavonol glycoside quantified, while syringetin 3-glucoside was not determined. Mikulic-Petkovsek et al. (2015) reported that flavonols were only present in small amounts in Ribes species ranging from $5 \%$ to $11 \%$ of total analyzed phenolic compounds and flavonols ranged from 36.12 to $53.94 \mathrm{mg} / \mathrm{kg}$ fw in Jostaberry.

It is difficult to make a direct comparison of bioactive compounds found in our research and those reported by other authors in Ribes species, since growing conditions, genotype, species, cultivar, fruit maturity, agro techniques, climatic factors, geographic region, and different extraction methods may affect the composition and concentration of phenolic compounds in these fruits (Strack, 1997; Tabart et al., 2006; Rubinskiene et al., 2005; Kellogg et al., 2010; Vagiri et al., 2013; Mikulic-Petkovsek et al., 2013). The data obtained from fruits, all in the same field and therefore cultivated in the same conditions, have confirmed that the genome and cultivar is the principal factor that determines the contents and compositions of phenolic compounds (Plessi et al., 2007).

The results of our study indicate that Ribes species, especially black currants are an exceptionally rich source of phenolic compounds and presents a nutritionally rich and healthy fruit. In all black currant cultivars cyanidin 3,5-di-O-glucoside, delphinidin 3-O-rutinoside, and cyanidin 3-O-rutinoside were the predominant anthocyanin compounds, respectively and in red currants only cyanidin 3-O-sambubioside and cyanidin 3-O-rutinoside were detected. In almost all Ribes species fruits quercetin 3-O-rutinoside was the major flavonol glycoside and epigallocatechin found as the dominant flavanol compound. It was found that black currants had the highest bioactive properties. Among the black currants, Rosenthal cultivar had the highest amounts of total phenols and antioxidant capacity levels, whereas Boskoop Giant cultivar characterized with the best content of anthocyanins and bioaccessible total phenolic content.

The wide cultivar of the phenolic compounds and values of antioxidant capacities found in the studied Ribes species fruit and implies their potential beneficial effects for human health. The obtained data can be used to encourage people to consume more these healthy fruits and can be also used further studies aimed at introducing promising varieties for cultivation.

\section{Acknowledgements}

The authors would like to thank TAGEM (General Directorate of Agricultural Research and Policies, Turkey) for financially support this research project (Project No: 17/A03/P06/139). Additionally, thanks are given to Central Research Institute of Food and Feed Control for technical support. This study is a part of $\mathrm{PhD}$ thesis of the first author (H. E. KARAAGAC).

\section{References}

Anttonen, M. J., \& Karjalainen, R. O. (2006). High-performance liquid chromatography analysis of black currant (Ribes nigrum L.) fruit phenolics grown either conventionally or organically. Journal of Agricultural and Food Chemistry, 54(20), 7530-7538. http://dx.doi. org/10.1021/jf0615350. PMid:17002418.

Apak, R., Guçlu, K., Ozyurek, M., \& Karademir, S. E. (2004). Novel total antioxidant capacity index for dietary polyphenols and vitamins $\mathrm{C}$ and $\mathrm{E}$, using their cupric ion reducing capability in the presence of neocuproine: CUPRAC method. Journal of Agricultural and Food Chemistry, 52(26), 7970-7981. http://dx.doi.org/10.1021/jf048741x. PMid:15612784.

Bordonaba, J. G., \& Terry, L. A. (2008). Biochemical profiling and chemometric analysis of seventeen UK-grown black currant cultivars. Journal of Agricultural and Food Chemistry, 56(16), 7422-7430. http://dx.doi.org/10.1021/jf8009377. PMid:18642846.

Borges, G., Degeneve, A., Mullen, W., \& Crozier, A. (2010). Identification of flavonoid and phenolic antioxidants in black currants, blueberries, raspberries, red currants, and cranberries. Journal of Agricultural and Food Chemistry, 58(7), 3901-3909. http://dx.doi.org/10.1021/ jf902263n. PMid:20000747.

Brand-Williams, W., Cuvelier, M. E., \& Berset, C. (1995). Use of a free radical method to evaluate antioxidant activity. LebensmittelWissenschaft + Technologie, 28(1), 25-30. http://dx.doi.org/10.1016/ S0023-6438(95)80008-5.

Chiang, C. J., Kadouh, H., \& Zhou, K. (2013). Phenolic compounds and antioxidant properties of gooseberry as affected by in vitro digestion. Lebensmittel-Wissenschaft + Technologie, 51(2), 417-422. http://dx.doi.org/10.1016/j.lwt.2012.11.014.

Contessa, C., Mellano, M. G., Beccaro, G., Giusiano, A., \& Botta, R. (2013). Total antioxidant capacity and total phenolic and anthocyanin contents in fruit species grown in Northwest Italy. Scientia Horticulturae, 160, 351-357. http://dx.doi.org/10.1016/j. scienta.2013.06.019.

Djordjević, B., Rakonjac, V., Fotirić Akšić, M., Šavikin, K., \& Vulić, T. (2014). Pomological and biochemical characterization of European 
currant berry (Ribes sp.) cultivars. Scientia Horticulturae, 165, 156162. http://dx.doi.org/10.1016/j.scienta.2013.11.014.

Djordjević, B., Savikin, K., Zdunic, G., Jankovic, T., Vulic, T., Oparnica, C., \& Radivojevic, D. (2010). Biochemical properties of red currant varieties in relation to storage. Plant Foods for Human Nutrition, 65(4), 326-332. http://dx.doi.org/10.1007/s11130-010-0195-z. PMid:21046468.

Ehala, S., Vaher, M., \& Kaljurand, M. (2005). Characterization of phenolic profiles of northern European berries by capillary electrophoresis and determination of their antioxidant activity. Journal of Agricultural and Food Chemistry, 53(16), 6484-6490. http://dx.doi.org/10.1021/ jf050397w. PMid:16076138.

Gavrilova, V., Kajdzanoska, M., Gjamovski, V., \& Stefova, M. (2011). Separation, characterization and quantification of phenolic compounds in blueberries and red and black currants by HPLC-DAD-ESI-MS. Journal of Agricultural and Food Chemistry, 59(8), 4009-4018. http:// dx.doi.org/10.1021/jf104565y. PMid:21401099.

Giusti, M. M., \& Wrolstad, E. (2001). Anthocyanins. In E. Wrolstad (Ed.), Characterization and measurement with UV-visible spectroscopy: current protocols in food analytical chemistry. New York: Wiley.

Justesen, U., Knuthsen, P., \& Leth, T. (1998). Quantitative analysis of flavonols, flavones, and flavanones in fruits, vegetables and beverages by high-performance liquid chromatography with photodiode array and mass spectrometric detection. Journal of Chromatography. A, 799(1-2), 101-110. http://dx.doi.org/10.1016/S0021-9673(97)010613. PMid:9550103.

Kellogg, J., Wang, J., Flint, C., Ribnicky, D., Kuhn, P., De Mejia, E. G., Raskin, I., \& Lila, M. A. (2010). Alaskan wild berry resources and human health under the cloud of climate change. Journal of Agricultural and Food Chemistry, 58(7), 3884-3900. http://dx.doi. org/10.1021/jf902693r. PMid:20025229.

Kim, D. O., Heo, H. J., Kim, Y. K., Yang, H. S., \& Lee, C. Y. (2005). Sweet and sour cherry phenolics and their protective effects on neuronal cells. Journal of Agricultural and Food Chemistry, 53(26), 9921-9927. http://dx.doi.org/10.1021/jf0518599. PMid:16366675.

Manach, C., Scalbert, A., Morand, C., Remesy, C., \& Jimenez, L. (2004). Polyphenols: Food sources and bioavailability. The American Journal of Clinical Nutrition, 79(5), 727-747. http://dx.doi.org/10.1093/ ajcn/79.5.727. PMid:15113710.

Mattila, P. H., Hellström, J., Karhu, S., Pihlava, J. M., \& Veteläinen, M. (2016). High variability in flavonoid contents and composition between different North-European currant (Ribes spp.) varieties. Food Chemistry, 204, 14-20. http://dx.doi.org/10.1016/j.foodchem.2016.02.056. PMid:26988470.

Mikulic-Petkovsek, M., Koron, D., \& Veberic, R. (2016). Quality parameters of currant berries from three different clusterpositions. Scientia Horticulturae, 210, 188-196. http://dx.doi.org/10.1016/j. scienta.2016.07.030.

Mikulic-Petkovsek, M., Rescic, J., Schmitzer, V., Stampar, F., Slatnar, A., Koron, D., \& Veberic, R. (2015). Changes in fruit quality parameters of four Ribes species during ripening. Food Chemistry, 173, 363-374. http://dx.doi.org/10.1016/j.foodchem.2014.10.011. PMid:25466034.

Mikulic-Petkovsek, M., Slatnar, A., Schmitzer, V., Stampar, F., Veberic, R., \& Koron, D. (2013). Chemical profile of black currant fruit modified by different degree of infection with black currant leaf spot. Scientia Horticulturae, 150, 399-409. http://dx.doi.org/10.1016/j. scienta.2012.11.038.

Mikulic-Petkovsek, M., Slatnar, A., Stampar, F., \& Veberic, R. (2012). HPLC-MSn identification and quantification of flavonol glycosides in 28 wild and cultivated berry species. Food Chemistry, 135(4),
2138-2146. http://dx.doi.org/10.1016/j.foodchem.2012.06.115. PMid:22980782.

Minekus, M., Alminger, M., Alvito, P., Ballance, S., Bohn, T., Bourlieu, C., Carrière, F., Boutrou, R., Corredig, M., Dupont, D., Dufour, C., Egger, L., Golding, M., Karakaya, S., Kirkhus, B., Le Feunteun, S., Lesmes, U., Macierzanka, A., Mackie, A., Marze, S., McClements, D. J., Ménard, O., Recio, I., Santos, C. N., Singh, R. P., Vegarud, G. E., Wickham, M. S. J., Weitschies, W., \& Brodkorb, A. (2014). A standardised static in vitro digestion method suitable for food: an international consensus. Food \& Function, 5(6), 1113-1124. http:// dx.doi.org/10.1039/C3FO60702J. PMid:24803111.

Nour, V., Stampar, F., Veberic, R., \& Jakopic, J. (2013). Anthocyanins profile, total phenolics and antioxidant activity of black currant ethanolic extracts as influenced by genotype and ethanol concentration. Food Chemistry, 141(2), 961-966. http://dx.doi.org/10.1016/j. foodchem.2013.03.105. PMid:23790874.

Pantelidis, G. E., Vasilakakis, M., Manganaris, G. A., \& Diamantidis, G. R. (2007). Antioxidant capacity, phenol, anthocyanin and ascorbic acid contents in raspberries, blackberries, red currants, gooseberries and Cornelian cherries. Food Chemistry, 102(3), 777-783. http:// dx.doi.org/10.1016/j.foodchem.2006.06.021.

Paunović, S. M., Mašković, P., Nikolić, M., \& Miletić, R. (2017). Bioactive compounds and antimicrobial activity of black currant (Ribes nigrum L.) berries and leaves extract obtained by different soil management system. Scientia Horticulturae, 222, 69-75. http:// dx.doi.org/10.1016/j.scienta.2017.05.015.

Plessi, M., Bertelli, D., \& Albasini, A. (2007). Distribution of metals and phenolic compounds as a criterion to evaluate variety of berries and related jams. Food Chemistry, 100(1), 419-427. http://dx.doi. org/10.1016/j.foodchem.2005.09.018.

Rubinskiene, M., Jasutiene, I., Venskutonis, P. R., \& Viskelis, P. (2005). HPLC determination of the composition and stability of blackcurrant anthocyanins. Journal of Chromatographic Science, 43(9), 478-482. http://dx.doi.org/10.1093/chromsci/43.9.478. PMid:16212794.

Singleton, V. L., Orthofer, R., \& Lamuela-Raventos, R. M. (1999). Analysis of total polyphenols and other oxidation substrates and antioxidants by means of Folin-Ciocalteu reagent. Methods in Enzymology, 299, 152-178. http://dx.doi.org/10.1016/S0076-6879(99)99017-1.

Slimestad, R., \& Solheim, H. (2002). Anthocyanins from black currants (Ribes nigrum L.). Journal of Agricultural and Food Chemistry, 50(11), 3228-3231. http://dx.doi.org/10.1021/jf011581u. PMid:12009991.

Strack, D. (1997). Phenolic metabolism. In P. M. Dey \& J. B. Harborne (Eds.), Plant biochemistry. London: Academic Press. http://dx.doi. org/10.1016/B978-012214674-9/50011-4.

Tabart, J., Kevers, C., Evers, E., \& Dommes, J. (2011). Ascorbic acid, phenolic acid, flavonoid, and carotenoid profiles of selected extracts from Ribes nigrum. Journal of Agricultural and Food Chemistry, 59(9), 4763-4770. http://dx.doi.org/10.1021/jf104445c. PMid:21417457.

Tabart, J., Kevers, C., Pincemail, J., Defraigne, J. O., \& Dommes, J. (2006). Antioxidant capacity of black currant varies with organ, season, and cultivar. Journal of Agricultural and Food Chemistry, 54(17), 6271-6276. http://dx.doi.org/10.1021/jf061112y. PMid:16910719.

Vagiri, M., Ekholm, A., Oberg, E., Johansson, E., Andersson, C. S., \& Rumpunen, K. (2013). Phenols and ascorbic acid in black currants (Ribes nigrum L.): variation due to genotype, location, and year. Journal of Agricultural and Food Chemistry, 61(39), 9298-9306. http://dx.doi.org/10.1021/jf402891s. PMid:24011264.

Vitali, D., Dragojević, I. V., \& Šebečić, B. (2009). Effects of incorporation of integral raw materials and dietary fibre on the selected nutritional and functional properties of biscuits. Food Chemistry, 114(4), 14621469. http://dx.doi.org/10.1016/j.foodchem.2008.11.032. 
Woznicki, T. L., Heide, O. M., Sønsteby, A., Wold, A. B., \& Remberg, S. F. (2015). Effects of controlled post-flowering temperature and daylength onchemical composition of four black currant (Ribes nigrum L.) cultivars of contrasting origin. Scientia Horticulturae, 197, 627-636. http://dx.doi.org/10.1016/j.scienta.2015.10.026.
Zafra-Stone, S., Yasmin, T., Bagchi, M., Chatterjee, A., Vinson, J. A., \& Bagchi, D. (2007). Berry anthocyanins as novel antioxidants in human health and disease prevention. Molecular Nutrition \& Food Research, 51(6), 675-683. http://dx.doi.org/10.1002/mnfr.200700002. PMid:17533652. 